\title{
Vaginal Stricture, CTCAE
}

National Cancer Institute

\section{Source}

National Cancer Institute. Vaginal Stricture, CT CAE. NCI Thesaurus. Code C143926.

A disorder characterized by a narrowing of the vaginal canal. 